\title{
Limitations of Near Edge X-Ray Absorption Fine Structure as a Tool for observing conduction bands in Chalcopyrite Solar Cell Heterojunctions
}

\section{Abstract}

A non-optimized interface band alignment in a heterojunction-based solar cell can have negative effects on the current and voltage characteristics of the resulting device. To evaluate the use of Near Edge X-ray Absorption Fine Structure spectroscopy (NEXAFS) as a means to measure the conduction band position, $\mathrm{Cu}(\mathrm{In}, \mathrm{Ga}) \mathrm{S}_{2}$ chalcopyrite thin film surfaces were investigated as these form the absorber layer in solar cells with the structure $\mathrm{ZnO} / \mathrm{Buffer} / \mathrm{Cu}(\mathrm{In}, \mathrm{Ga}) \mathrm{S}_{2} / \mathrm{Mo} /$ Glass. The composition dependence of the structure of the conduction bands of $\mathrm{CuIn}_{x} \mathrm{Ga}_{1-x} \mathrm{~S}_{2}$ has been revealed for $x=0,0.67$ and 1 with both hard and soft NEXAFS and the resulting changes in conduction band offset at the junction with the buffer layer discussed. A comprehensive study of the positions of the absorption edges of all elements was carried out and the development of the conduction band with Ga content was observed, also with respect to calculated densities of states.

\section{ARticle}

Valence and conduction band (VB, CB) alignments at heterojunctions play important roles in the functionality of semiconductor heterojunction devices such as the chalcopyrite thin layer solar cell based on the structure $\mathrm{n}^{+}-\mathrm{ZnO} / \mathrm{i}-\mathrm{ZnO} / \mathrm{Buffer} / \mathrm{Cu}(\mathrm{In}, \mathrm{Ga}) \mathrm{S}_{2} / \mathrm{Mo} /$ glass and others: Buffer $/ \mathrm{Cu}(\mathrm{In}, \mathrm{Ga}) \mathrm{Se}_{2}$, Buffer/CdTe or a-Si/c-Si $[1,2,3]$. The sulfide-based device with a CdS buffer layer has reached an efficiency of about 13\% [4]. More specifically, whether or not the $\mathrm{CB}$ offset at the buffer/absorber junction is optimized, that is, whether the $\mathrm{CB}$ edge of the absorber is closer to the Fermi level than the $\mathrm{CB}$ of the buffer (spike) or vice versa (cliff) can influence the current-voltage characteristics of the device. The reduced effective band gap of the cliff configuration can limit the photovoltage while the position in the junction where $\mathrm{p}=\mathrm{n}$ moves closer to the defect-rich buffer/absorber interface leading to increased charge carrier recombination [1]. 
Knowledge of these offsets is, therefore, critical to understanding the performance of the resulting solar cell. While the VB offset, $\Delta E_{V B}$, can be determined with established methods, such as combined XPS/UPS [5, 6] or Constant Final State Yield Spectroscopy [7], a determination of $\mathrm{CB}$ edge positions and offsets, $\Delta E_{C B}$, has proved more difficult. The most common method is simply the assumption that the CB minimum is the energy of the VB plus the band gap. However, the determination of the surface band gap, which is relevant for the band offset, is more involved. Two of the main methods for the direct determination of the CB minimum are inverse photoelectron spectroscopy (IPES) and Near Edge X-ray Absorption Fine Structure (NEXAFS). They have given reliable results in some situations $[8,9,10,11]$, although both have unresolved difficulties and the results must be carefully analyzed. IPES requires high intensity electron irradiation of the sample which often leads to charging of less conductive materials. In the case of NEXAFS these include transition probabilities, spectrum broadening and excitonic or core-hole effects. The latter may cause shifts in the measured position of the absorption edges which do not correspond to the ground state of the material. This is because the position of the absorption edge in NEXAFS represents the energy difference between the initial state (core level) and the final empty state (conduction band) in the material's excited state. The attraction between the core-hole and the excited electron may make the energy difference between the core level and conduction band state appear artificially smaller than it is in the ground state of the material. Also, because the absorption edge represents an energy difference, the energy of the initial state (core level) must be considered to determine whether differences in binding energy could influence the calculated energy of the final conduction band state. Here, while considering only the position of the absorption edge, we assume at first a constant initial state (core level binding) energy, although in several cases we explicitly consider specific measured binding energies. This assumption of constant binding energies will have immediate relevance when considering differences between the $\mathrm{CuInS}_{2}$ and $\mathrm{Cu}(\mathrm{In}, \mathrm{Ga}) \mathrm{S}_{2}$ samples because they are stoichiometrically similar.

Complicating matters still further, a junction, by its very existence, implies a buried interface which is often difficult to examine using surface sensitive methods and important 
processes such as chemical shifts during junction formation must often be neglected in order to determine an offset value $[12,13,14]$.

As will be seen, only a semi-quantitative assessment of the NEXAFS measurements was made because of the difficulties in establishing an absolute calibration of the energy scales. We, therefore, keep our focus on examining the validity of NEXAFS as a tool for monitoring $\mathrm{CB}$ edge positions rather than an actual determination of $\Delta E_{C B}$. For this reason bare solar cell grade $\mathrm{CuIn}_{x} \mathrm{Ga}_{1-x} \mathrm{~S}_{2}(x=0,0.67$ and 1$)$ or "CIGS" layers grown by rapid thermal processing (RTP) [15] were studied after being etched in a standard solution of $5 \% \mathrm{KCN}$ for three minutes to remove the secondary $\mathrm{Cu}_{x} \mathrm{~S}$ phase [16]. Inferences about the junction with CdS are then made by using the results of other measurements and we remain mainly on qualitative footing.

The samples were loaded into the vacuum chamber $\left(\sim 10^{-9} \mathrm{mbar}\right)$ immediately after preparation and no subsequent sputtering of surfaces was undertaken in order to clean them. The measurements were performed at the BESSY II synchrotron in Berlin, Germany. The absorption edges in the soft X-ray regime (50-2000 eV) were made on the Optics beam line with the SurICat end station and detected using total electron yield (sample current) mode so that the information depth exceeded the 1-4 nm reached with XPS. Estimates of the NEXAFS information depths reach into the tens of nm and, like the information depths in PES, are dependent on electron kinetic energy and vary between absorption edges. The structure in the mirror current from the refocusing mirror of the beamline $\left(\mathrm{I}_{0}\right)$ caused by X-ray absorption from elements on the mirror was used as a reference so that all of the spectra from each individual element enjoyed a common, albeit not necessarily absolute, energy scale and real shifts between the absorption edges can be considered. Although the elements on the mirror are unknown, their absorption features occur at energies which do not change during the time scale of the measurements. 
The hard X-ray edges (2000-10,000 eV) were measured at the KMC-1 beam line with the HIKE end station using X-ray emission with a Bruker fluorescence detector. The information depths reach into the $\mu \mathrm{m}$ range and are much less surface sensitive than the soft X-ray measurements. Because no refocusing optics exist and the analogue of the mirror current, the $\mathrm{N}_{2}$-ionization current, was not useful in calibrating the energy scale each edge was measured successively on each sample with a low scan count in order to avoid broadening or shifts of the absorption edge. This is caused by the monochromator not scanning the exact energy window with every sweep (monochromator reproducibility) and could not be corrected for on this beamline. While the resulting spectra are somewhat noisy, it again enables the comparison of the spectra on an energy scale common to all curves for each element.

In the analysis of NEXAFS spectra the definition of the "position" of the edge itself can be problematic and the literature provides several different methods of determining the position of the edge. These include fitting the the crest of the curve immediately after the absorption edge with the initial-state core level [9], extrapolation of the absorption edge to the background [10, 11], the maximum of its first derivative [17] as well as consideration of a fitted density of states calculation. In addition, the second derivative can also be used, although this is often in an attempt to differentiate two peaks separated by an energy close to that of the experimental resolution [18]. In more complex attempts, often reserved for EXAFS data, a polynomial background can be subtracted after which a spline fit is used for normalization [19].

In this study we have chosen to observe mainly overall shifts in the peaks which amounts to the position of the absorption edge at its half-maximum. This method gives us satisfactory trends and other factors must be clarified before the results would profit from any increased accuracy derived from the methods mentioned above. We also consider the position of peaks after the absorption edge as in [9] although we have not carried out in-depth fits using the measured core levels. 

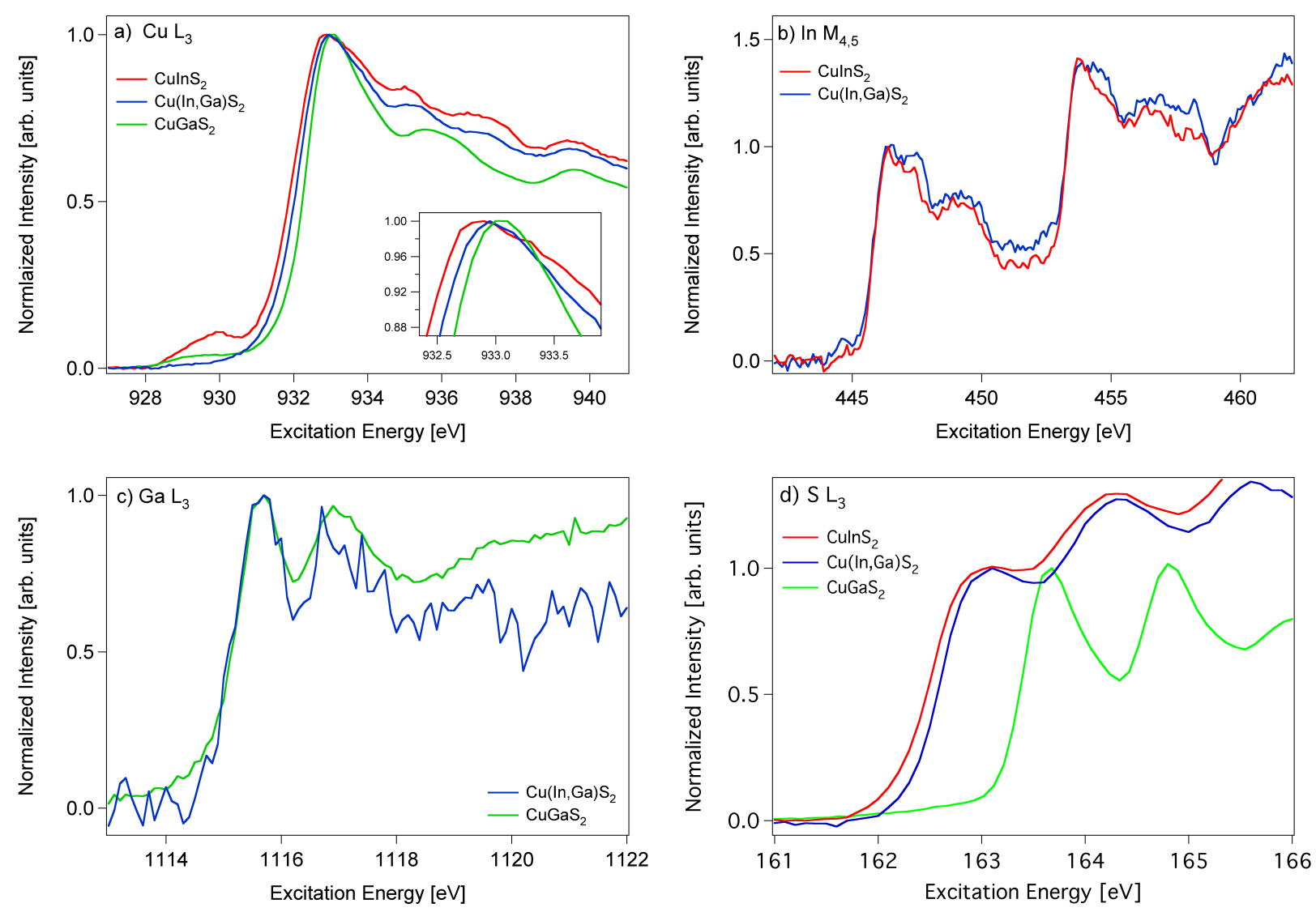

Figure 1. Absorption edges of a) $\mathrm{Cu} \mathrm{L}_{3}$, b) In $\mathrm{M}_{4,5}$, c) $\mathrm{Ga} \mathrm{L}_{3}$ and d) $\mathrm{S} \mathrm{L}_{3}$ from $\mathrm{CuInS}_{2}$ (red), $\mathrm{CuIn}_{0.67} \mathrm{Ga}_{0.33} \mathrm{~S}_{2}$ (blue) and $\mathrm{CuGaS}_{2}$ (green) measured with total electron yield. The energy scale for each set of curves is not absolute, but the edge positions are correct relative to one another, making shifts in the spectra correspond to real energy differences between the core level and the end state of the electron in the conduction bands of the three materials. Inset: the crests of the $\mathrm{CuL}_{3}$ spectra.

Fig. 1 shows the soft X-ray NEXAFS measurements on the $\mathrm{Cu} \mathrm{L}_{3}, \mathrm{In}_{4,5}, \mathrm{Ga} \mathrm{L}_{3}$ and $\mathrm{S} \mathrm{L}_{3}$ absorption edges from $\mathrm{CuInS}_{2}$ (red), $\mathrm{CuIn}_{0.67} \mathrm{Ga}_{0.33} \mathrm{~S}_{2}$ (blue) and $\mathrm{CuGaS}_{2}$ (green). Although the edges are shown before the subtraction of the corresponding core level binding energies, the subtraction did not always change the results and will be considered in cases where the subtraction led to significant differences. The shifts seen in fig. 1 correspond then, at least qualitatively, to shifts in the CB states of the CIGS with varying $[\mathrm{Ga}] /[\mathrm{In}+\mathrm{Ga}]$ ratios. It can immediately be seen that $\mathrm{Cu} \mathrm{L}_{3}$ (see inset) and $\mathrm{S} \mathrm{L}_{3}$ edges are shifted and correspond to an opening of the CIGS band gap with increasing Ga concentration while the In $\mathrm{M}_{4,5}$ and $\mathrm{Ga} \mathrm{L}_{3}$ edges display no shift (the noisy CIGS spectrum from Ga is due to the low surface Ga content of $\sim 8 \%$ ). It is evident that several measurements of different absorption edges 
are needed in order to understand the development of the CB and one measurement, while not incorrect, may not illustrate fully the differences between the systems.

Before focusing on the shifts in fig. 1 a) and d) it is helpful to consider the VB edge positions (measured with UPS, not shown) and bulk band gaps, $\mathrm{E}_{g}$, of CIGS shown in table 1 for an estimate of where the $\mathrm{CB}$ edges of the three materials may be expected. The $\mathrm{E}_{g}$ values do not necessarily reflect values for solar cell-grade surfaces due to stoichiometric gradients which may cause differences between bulk and surface characteristics [20, 21]. The values in the table show a CIS CB edge $0.8 \mathrm{eV}$ above the Fermi Level with the $\mathrm{CuIn}_{0.67} \mathrm{Ga}_{0.33} \mathrm{~S}_{2} \mathrm{CB}$ edge $0.1 \mathrm{eV}$ further up and the CGS $1.1 \mathrm{eV}$ beyond that.

TABLE 1. Measured valence band edge positions (energy below Fermi Level, $\mathrm{E}_{f}$ ), bulk band gaps $\left(\mathrm{E}_{g}\right)$ and resulting estimated conduction band edge positions (energy above $\mathrm{E}_{f}$ ) for $\mathrm{CuInS}_{2}, \mathrm{CuIn}_{0.67} \mathrm{Ga}_{0.33} \mathrm{~S}_{2}$ and $\mathrm{CuGaS}_{2}$.

\begin{tabular}{|c|c|c|c|}
\hline Sample & VB Edge $\left(\mathrm{E}_{f}-\mathrm{E}_{V B}\right)$ & Band Gap & CB Edge $\left(\mathrm{E}_{f}+\mathrm{E}_{C B}\right)$ \\
\hline $\mathrm{CuInS}_{2}$ & $0.7 \mathrm{eV}$ & $1.5 \mathrm{eV}$ & $0.8 \mathrm{eV}$ \\
\hline $\mathrm{CuIn}_{0.67} \mathrm{Ga}_{0.33} \mathrm{~S}_{2}$ & $0.7 \mathrm{eV}$ & $1.6 \mathrm{eV}$ & $0.9 \mathrm{eV}$ \\
\hline $\mathrm{CuGaS}_{2}$ & $0.4 \mathrm{eV}$ & $2.4 \mathrm{eV}$ & $2.0 \mathrm{eV}$ \\
\hline
\end{tabular}

Fig. 1 a) shows the $\mathrm{Cu} \mathrm{L}_{3}$ edges with a crest shift between the CIS and CIGS measurements of $150 \mathrm{meV} \pm 30 \mathrm{meV}$, similar to that found in table 1. Further supporting this as a real shift in the $\mathrm{CB}$ is the fact that the CIS and $\mathrm{CuIn}_{0.67} \mathrm{Ga}_{0.33} \mathrm{~S}_{2}$ crystal lattices are similar meaning other factors influencing the position of an absorption edge, such as excitonic effects, can be disregarded as they will be the same in both materials. The fine structure after the absorption edge is similar for CIS and CIGS showing a similarity of the conduction bands and further supports the similarities of both lattices. One exception is the characteristic drop in intensity in the CIGS spectrum just after the crest and is even more pronounced in the CGS sample. However, the fine structure of the CGS is different than the other two materials and the shift between CIGS and CGS is anomalously small, $0.25 \mathrm{eV}$, as compared to the $1.1 \mathrm{eV}$ expected from the bulk band gap estimation. The measurement itself may affect the position of the CGS $\mathrm{Cu} \mathrm{L}_{3}$ absorption edge differently than that of CIS and CIGS. Analysis after the subtraction of the $\mathrm{Cu} 2 \mathrm{p}_{3 / 2}$ core level binding energy (not shown) corrected this 
discrepancy somewhat so that the shift between CIGS and CGS was $0.40 \mathrm{eV}$ while leaving the shift between CIS and CIGS intact. Differences in the material properties at the differing information depths of the NEXAFS measurements and XPS measurements of the core levels could be partly responsible for the small shift between CIGS and CGS. Stoichiometric gradients in solar cell-grade chalcopyrite layers leading to different surface and bulk properties are well known $[20,21]$. In the case of the $\mathrm{Cu} \mathrm{L}_{3}$ measurement the rather high binding energy of the $\mathrm{Cu} 2 \mathrm{p}_{3 / 2}$ core level $(932 \mathrm{eV})$ leads to high resulting electron kinetic energy for the NEXAFS measurements and low electron kinetic energy for the XPS measurements (excitation energy $\mathrm{h} \nu=1150 \mathrm{eV}$ ) and, therefore, differing information depths of the measurements. If the width of the depletion region on the surface of the CGS samples is different from that on CIS and CIGS this could lead to discrepancies in the observed shifts. Also noticeable is the pre-edge feature in the CIS spectrum which is due to a surface phase with a $\mathrm{Cu}$ d-9 electronic structure and is not from the CIS [22].

The $\mathrm{S} \mathrm{L}_{3}$ measurements differ from $\mathrm{Cu} \mathrm{L}_{3}$ due to the relatively shallow $\mathrm{S} 2 \mathrm{p}$ doublet $(181 \mathrm{eV})$. Here, with the same excitation energy as before $(\mathrm{h} \nu=1150 \mathrm{eV})$, the resulting electron kinetic energy in XPS will be $750 \mathrm{eV}$ higher than the $\mathrm{Cu} 2 \mathrm{p}$ electrons while the resulting kinetic energy in the NEXAFS measurements will be lower resulting in more similar information depths for the sulphur XPS and NEXAFS measurements. Here the shift between the CIS and CIGS is $0.1 \mathrm{eV}$ while between CIGS and CGS is $0.8 \mathrm{eV}$, slightly smaller than that expected from table 1 . The values were determined using the edge positions at the half-maximum because the crest positions were not unambiguous due to slight broadening. One can also consider the $\mathrm{S} \mathrm{L}_{3}$ edges after the subtraction of the $\mathrm{S} 2 \mathrm{p}_{3 / 2}$ core level binding energies (fig. 2).

Perhaps due to the similarity in information depths of the XPS and NEXAFS measurements, the position of zero binding energy (Fermi Level) is plausible. In the case of the $\mathrm{Cu}$ $\mathrm{L}_{3}$ edges after the subtraction of the $\mathrm{Cu} 2 \mathrm{p}_{3 / 2}$ core level binding energy, all edges crossed (were to the left of) zero binding energy, indicating a discrepancy between the XPS and NEXAFS measurements. The positions of the $\mathrm{S} \mathrm{L}_{3}$ absorption edges when compared to 


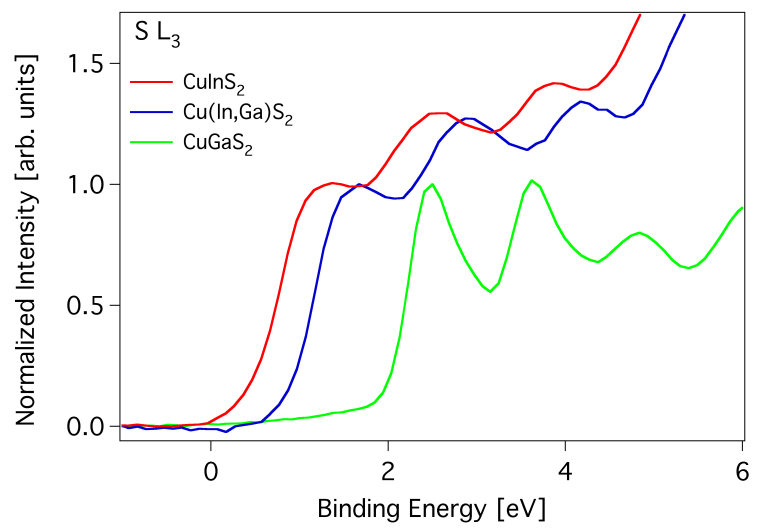

Figure 2. $\mathrm{S} \mathrm{L}_{3}$ absorption edges from $\mathrm{CuInS}_{2}$ (red), $\mathrm{CuIn}_{0.67} \mathrm{Ga}_{0.33} \mathrm{~S}_{2}$ (blue) and $\mathrm{CuGaS}_{2}$ (green) after the subtraction of the measured $\mathrm{S} 2 \mathrm{p}_{3 / 2}$ core level binding energies. The large shift between CIS and CGS is reflective of the large change in band gap between the two materials.

zero binding energy in fig. 2 are $0.7 \mathrm{eV}, 1.1 \mathrm{eV}$ and $2.2 \mathrm{eV}$ for CIS, CIGS and CGS, respectively. The shifts differ somewhat from those expected by the bulk band gap estimation with the total spread from CIS to CGS of $1.5 \mathrm{eV}$ somewhat larger than that found in table 1. However, these values still reflect the large difference in the band gaps of the two materials.

The $\mathrm{S} \mathrm{L}_{3}$ measurements are, therefore, in better agreement with the bulk band gap estimations than the $\mathrm{Cu} \mathrm{L}_{3}$ measurements. Apart from considerations about the information depths, this could be due to similarities of the electronic states of $\mathrm{S}$ in the CIS, CIGS and CGS lattices so that effects of the measurement (excitonic, etc.) are the same in each material. However, differences in the fine structure of the measurement after the absorption edge are present. Recent theoretical calculations have also shown that the position of the S (Se) atoms in the CIGS (CIGSe) lattice has a large effect on the final electronic characteristics of the material [23]. It may not be surprising then, that the $\mathrm{S}$ measurements contain the most relevant and realistic electronic information about the change in position of the $\mathrm{CB}$ edge of all the soft X-ray measurements.

The hard X-ray measurements are shown in fig. 3. In contrast to fig. 1, the only set of curves displaying a shift is $\mathrm{S}$ in fig. $3 \mathrm{~d}$ ). It should also be kept in mind, that this set of 
figures is not burdened by surface effects, as emission is more bulk sensitive.
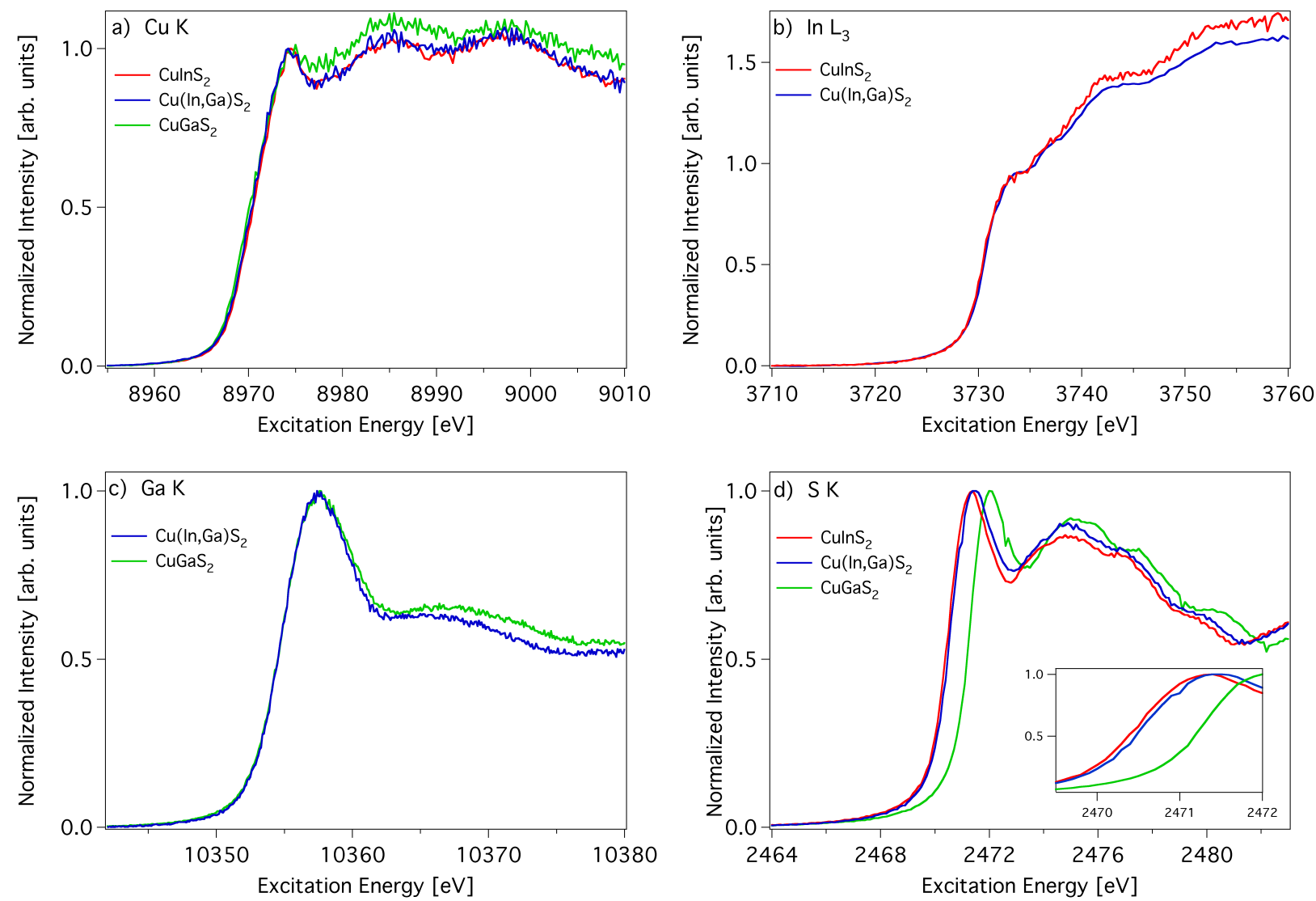

Figure 3. Absorption edges of a) $\mathrm{Cu} \mathrm{K}$, b) In $\mathrm{L}_{3}$, c) Ga K and d) S K from $\mathrm{CuInS}_{2}$ (red), $\mathrm{CuIn}_{0.67} \mathrm{Ga}_{0.33} \mathrm{~S}_{2}$ (blue) and $\mathrm{CuGaS}_{2}$ (green) measured with Xray fluorescence. The energy scale for each set of curves is not absolute, but the edge positions are correct relative to one another, making shifts in the spectra correspond to real energy differences between the core level and the end state of the electron in the conduction bands of the three materials. Inset: the crests of the S K spectra.

Fig. 3 a) shows the $\mathrm{Cu} \mathrm{K}$ edge with no significant shift between the crests of the curves. This measurement is sensitive to p-type states, whereas the $\mathrm{L}_{3}$ edges, which displayed a shift, are sensitive to s- and d-type partial density of states (DOS) meaning that the lack of shift here is not necessarily a contradiction. The fine structure after the edge is, unlike the $\mathrm{L}_{3}$ measurements, similar in all three K edge curves.

The In $\mathrm{L}_{3}$ edges shown in fig. $3 \mathrm{~b}$ ) ( $\mathrm{s}$ - and d-DOS) display no shift as was the case with the In $\mathrm{M}_{4,5}$ edges (p-type DOS) in fig. $1 \mathrm{~b}$ ). Similarly, the Ga K (d-DOS) edges shown in 
fig. $3 \mathrm{c}$ ) display no shift as was the case with the $\mathrm{G} \mathrm{L}_{3}$ (s- and d-DOS) edges in fig. $1 \mathrm{c}$ ).

Finally, the S K edges are displayed in fig. $3 \mathrm{~d}$ ) with an inset close-up of the crests. Here a shift is also found, nearly equal whether one uses the crests or the edges positions at halfmaximum. Between CIS and CIGS the shift is $0.1 \mathrm{eV}$ and between CIGS and CGS $0.7 \mathrm{eV}$, the latter being somewhat smaller than expected. The S measurements also contain the most relevant, realistic electronic information about the change in position of the $\mathrm{CB}$ edge among the hard X-ray measurements. In addition, as with the $\mathrm{Cu} \mathrm{K}$ and $\mathrm{L}_{3}$ measurements, the fine structure of the three hard X-ray curves for S is more similar than that of the soft X-ray curves.

The comparison between the absorption edges in the hard and soft X-ray regimes addresses two further important questions about the NEXAFS measurements. With the exception of $\mathrm{Cu}$, the behavior of the absorption edges was the same for each element in both regimes and indicates, firstly, the binding energy of the core level plays only a limited role in determining the position of the edge. If the core level binding energy was indeed responsible for the position of the edge in one regime, we would expect it also to play a role in the other regime, but with a different magnitude. Secondly, the vast difference in information depth between the two regimes shows that any change in information depth in a single energy window, being smaller by comparison, will not drastically alter the position of any one edge.

According to preliminary DOS calculations done with the Stuttgart TB-LMTO program (not shown) $[24,25,26]$ as well as in other literature sources [27, 28], it can be seen that In and Ga s-states dominate the CB edge. Fig. 1 c) $\left(\mathrm{Ga} \mathrm{L}_{3}\right)$ and fig. 3 b) ( $\left.\mathrm{In} \mathrm{L}_{3}\right)$ show, however, no shift, although these measurements are sensitive to exactly these states. Furthermore, the $\mathrm{Cu}$-s and d states make only a small contribution to the CB edge, although a shift was seen in fig. $1 \mathrm{a})\left(\mathrm{Cu} \mathrm{L}_{3}\right)$. In contrast, the $\mathrm{Cu}$-p states have a contribution similar to the $\mathrm{s}$ and d states, but no shift was seen in fig 3 a) $(\mathrm{Cu} \mathrm{K})$. 
The S p-type contribution to the CB edge is large and one sees a shift in fig. $3 \mathrm{~d})(\mathrm{S} \mathrm{K})$, however, a similar shift is seen in fig. $1 \mathrm{~d})\left(\mathrm{S} \mathrm{L}_{3}\right)$, although the S-s and d states make a much smaller contribution.

Therefore, it can be concluded that in the case of $\mathrm{Cu}(\mathrm{In}, \mathrm{Ga}) \mathrm{S}_{2}$ although information about $\mathrm{CB}$ edge position is contained in a full set of NEXAFS spectra (all elements, all pertinent edges), this information is not contained in each set of element-specific spectra. Even if certain elements have a large contribution to the CB edge, the position of the absorption edge can still be independent of stoichiometry. This leads to the idea that there is a local electronic environment around each atom which can be independent of stoichiometry with the optically measurable band gap of the material only becoming evident at macroscopic levels. For In and Ga the local electronics do not depend strongly on the stoichiometry of the sample, while for $\mathrm{S}$ the dependence is critical and corresponds to theoretical calculations. Although $\mathrm{Cu}$ the anomalous behavior of the $\mathrm{Cu}$ edge, that is, the shift in the $\mathrm{L}_{3}$ edges and absence thereof in the K-edge, cannot yet be fully interpreted, it can be expected that the changing environment around the $\mathrm{Cu}$ atoms with stoichiometry (exchange of the group III elements In and $\mathrm{Ga}$ ) will lead to a change in some of its electronic states.

Because of the important differences between the ground and excited states in X-ray spectroscopic experiments [29, 30], we mention again that NEXAFS probes the electronic positions of the samples in an excited state with a core hole and that the position of the absorption edge represents the difference between the core level and conduction band state in the excited state of the material. The features found in figs. 1 and 3 maybe be effected by excitons which mask the true positions of the CB edge. Therefore, this phenomena must also be more carefully investigated before we can assign the positions of the absorptions edges to a local electronic structure with certainty or make final quantitative assessments.

Nevertheless, the NEXAFS measurements show that the CB offset, $\Delta E_{C B}$ increases in the CIGS/CdS junction with increasing Ga content because the opening of the CIGS band gap increases the potential energy difference between the CIGS and CdS conduction bands. 
The increase between CIS and $\mathrm{CuIn}_{0.67} \mathrm{Ga}_{0.33} \mathrm{~S}_{2}$ is between 0.2 and $0.3 \mathrm{eV}$ while the shift between CIGS and CGS is $\sim 1 \mathrm{eV}$ where we, again, assume constant core level binding energies and the absence of excitonic effects for these quantitative numbers. While differing binding energies may alter the interpretation given here, the measured binding energies discussed above would only have the effect of changing the magnitude of the increase in the band offset between CIGS and CdS. Although this increase exacerbates the CB offset between the two materials with increased Ga content and leaves the effective band gap of the junction the same [1], the solar cell shows the best efficiency at Ga concentrations similar to that studied here $\left([\mathrm{Ga}]_{\text {Surface }}=\sim 8 \%\right)[31]$. This means that the addition of Ga improves the heterojunction by a different means, such as a reduction of interface defects.

NEXAFS has been shown to be an effective, if still not fully understood, tool for the investigation of the stoichiometric-dependent development of semiconductor conduction bands. This can lead to the development of a synchrotron-based method for determining CB positions which is complementary to IPES. Further research is underway to understand what information about the observed system is contained in each set of curves and how this can be used to explain the behavior of the conduction band with changing stoichiometry.

\section{ACKNowledgements}

Financial support was provided by the German Bundesministerium für Umwelt, support code 0327589B (KD-CIS).

\section{REFERENCES}

[1] R. Klenk, Thin Solid Films 387, 135-140 (2001)

[2] L. Korte, A. Laades, M. Schmidt, J. Non-Cryst. Sol. 352, 1217-1220 (2006)

[3] W. Jaegermann, A. Klein, T. Mayer, Advance Materials 21, 4196-4206 (2009)

[4] S. Merdes, B. Johnson, J. Klaer, R. Klenk, I. Lauermann, R. Mainz, A. Meeder, Thin-Film Compound Semiconductor Voltaics-2009. Book Series: Mater. Res. Soc. Symp. Proc. 1165, 179-184 (2010)

[5] Y. Hashimoto, K. Takeuchi, K. Ito, Appl. Phys. Lett. 67, 980-982 (1995) 
[6] L. Weinhardt, O. Fuchs, D. Groß, G. Storch, E. Umbach, N. G. Dhere, A. A. Kadam, S. S. Kulkarni, C. Heske, Appl. Phys. Lett. 86, 062109-062111 (2005)

[7] L. Korte, M. Schmidt, J. Non-Cryst. Sol. 354, 2138-2143 (2008)

[8] Ch.-H. Fischer, M. Bär, Th. Glatzel, I. Lauermann, M.C. Lux-Steiner, Solar Energy Materials \& Solar Cells 90, 1471-1485 (2006)

[9] A. Sandell, B. Sanyal, L.E. Walle, P. Uvdal, A. Borg, J. Electron Spec. and Rel. Phen. 183, 1107-113 (2011)

[10] M. Bär, B.-A. Schubert, B. Marsen, S. Schorr, R.G. Wilks, L. Weinhardt, S. Pookpanratana, M. Blum, S. Krause, Y. Zhang, W. Yang, T. Unold, C. Heske, and H.-W. Schock, Phys. Rev. B 84, 035308 (2011)

[11] M. Bär, S. Nishiwaki, L. Weinhardt, S. Pookpanratana, O. Fuchs, M. Blum, W. Yang, J.D. Denlinger, W.N. Schafarman, C. Heske, Appl. Phys. Lett. 93, 244103 (2008)

[12] T. Schulmeyer, R. Kniese, R. Hunger, W. Jaegermann, M. Powalla, A. Klein, Thin Solid Films 451, 420-423 (2004)

[13] N.G. Dhere, A.A. Kadam, A.H. Jahagirdar, S.S. Kulkarni, L. Weinhardt, D. Groß, C. Heske, E. Umbach, J. Phys. Chem. Solids 66, 1872-1875 (2005)

[14] B. Johnson, L. Korte, T. Lußky, J. Klaer, I. Lauermann, J. Appl. Phys. 106, 073712 (2009)

[15] S. Siemer, J. Klaer, I. Luck, J. Bruns, R. Klenk, D. Bräunig, Solar Energy Materials and Solar Cells 67, 159-166 (2001)

[16] R. Klenk, J. Klaer, R. Scheer, M.Ch. Lux-Steiner, I. Luck, N. Meyer, U. Rühle, Thin Solid Films 480-481, 509-514 (2005)

[17] A.J. Berry, H. St.C. O’Niel, K.D. Jayasuriya, S.J. Campbell, G.J. Foran, Am. Min. 88, 967-977 (2003)

[18] V. Sikolenko, A. Sazonov, V. Efimov, V. Krivencov, N. Darowski, D. Vyalikh, J. Mag. Mag. Mat. 310, e181-e183 (2007)

[19] T.-C. Weng, G.S. Waldo, J.E. Penner-Hahn, J. Synch. Rad. 12, 506-510 (2005)

[20] H. Mönig, Ch.-H. Fischer, R. Caballero, C.A. Kaufmann, N. Allsop, M. Gorgoi, R. Klenk, H.-W. Schock, S. Lehmann, M.C. Lux-Steiner, I. Lauermann, Acta Materialia 57, 3645-3651 (2009)

[21] U. Rau, H.W. Schock, Appl. Phys. A 69, 131-147 (1999)

[22] M. Grioni, J.B. Goedkoop, R. Schoorl, F. M. F. de Groot, J.C. Fuggle, Phys. Rev. B 39, 1541-1545 (1989)

[23] J. Vidal, S. Botti, P. Olsson, J-F. Guillemoles, L. Reining, Phys. Rev. Lett. 104, 056401 (2010)

[24] O.K. Andersen and O. Jepsen, Phys. Rev. Lett. 53, 2571 (1984)

[25] O.K. Andersen, O. Jepsen, and D. Gloetzel, Highlights of condensed-Matter Theory, ed. F. Bassani, F. Fumi, and M.P. Tosi (North-Holland, New York, 1985)

[26] Further information and download of the Stuttgart TB-LMTO program available at http://www.fkf.mpg.de/anderson 
[27] R. Bacewicz, A. Wolska, K. Lawniczak-Jablonska, Ph. Sainctavit, J. Phys.: Condens. Matter 12, 7371$7379(2000)$

[28] A. Soni, V. Gupta, C.M. Arora, A. Dashora B.L. Ahuja, Solar Energy 84, 1481-1489 (2010)

[29] A. Nilsson, N. Mårtensson, Physica B 208-209, 19-22 (1995)

[30] A. Nilsson, J. Stöhr, T. Wiell, M. Alden, P. Bennich, N. Wassdahl, M. G. Samant, S. S. P. Parkin, Phys. Rev. B 54, 2917-2921 (1996)

[31] S. Merdes, R. Mainz, J. Klaer, A. Meeder, H. Rodriguez-Alvarez, H.W. Schock, M.Ch. Lux-Steiner, R. Klenk, Solar Energy Materials \& Solar Cells 95, 864-869 (2011) 